\title{
Quadratic Program on a Structured Nonconvex Set
}

\author{
Yi Xu $\mathbb{D}^{1}$ and Lili Han $\mathbb{D}^{2}$ \\ ${ }^{1}$ Mathematics Department, Southeast University, 2 Sipailou, Nanjing, Jiangsu Province 210096, China \\ ${ }^{2}$ School of Management Science, Qufu Normal University, Rizhao, Shandong 276800, China
}

Correspondence should be addressed to Yi Xu; yi.xu1983@hotmail.com

Received 25 September 2019; Revised 5 February 2020; Accepted 17 February 2020; Published 12 March 2020

Academic Editor: Gen Q. Xu

Copyright (C) 2020 Yi Xu and Lili Han. This is an open access article distributed under the Creative Commons Attribution License, which permits unrestricted use, distribution, and reproduction in any medium, provided the original work is properly cited.

In this paper, we focus on a special nonconvex quadratic program whose feasible set is a structured nonconvex set. To find an effective method to solve this nonconvex program, we construct a bilevel program, where the low-level program is a convex program while the upper-level program is a small-scale nonconvex program. Utilizing some properties of the bilevel program, we propose a new algorithm to solve this special quadratic program. Finally, numerical results show that our new method is effective and efficient.

\section{Introduction}

In this paper, we discuss the following structured quadratic program:

$$
\begin{array}{ll}
\text { inf } & x^{T} A x+2 a^{T} x \\
\text { s.t. } & x \in \Omega,
\end{array}
$$

where $x \in \mathbb{R}^{n}$, $S^{n \times n} \sum A>0, a \in \mathbb{R}^{n}$, and $\Omega \subset \mathbb{R}^{n}$ is a set.

Program (1) has been frequently exploited in optimization theory and practice [1-5]. If $\Omega$ is a closed convex set, program (1) is a convex program, which can be solved by many algorithms such as cutting plane method, projection method, and interior point method [1-3, 6-8]. However, in practice, program (1) is usually a nonconvex program since $\Omega$ is often a nonconvex set. As we know, a nonconvex program is difficult to solve since it is a NP-hard problem in general. However, there are still some special small-scale nonconvex problems which can be easily solved by some algorithms. For example, finding the roots of a continuous function $f(x)$ in $x \in[a, b] \subset \mathbb{R}$ has polynomial time computational complexity, although this problem is nonconvex. This motivates us to covert program (1) into a smallscale program.

Note that for a bounded closed set $\Omega$, by the Hein-Borel theorem, there exists a finite set $\Xi \subset \mathbb{R}^{n}$ such that $\Omega$ can be covered by

$$
\bigcup_{x_{k} \in \Xi}\left\{x \in \mathbb{R}^{n} \mid\left(x-x_{k}\right)^{T} B_{k}\left(x-x_{k}\right)+c_{k} \geq 0\right\}
$$

where $B_{k} \prec 0$ and $c_{k}>0$. This says that there exists a finite index set $\Gamma$ such that $\Omega$ is covered by

$$
\bigcup_{t \in \Gamma}\left\{x \in \mathbb{R}^{n} \mid x^{T} B(t) x+2 b(t)^{T} x+c(t) \geq 0\right\},
$$

where $B(t)<0$ and $c(t)-b(t)^{T} B(t)^{-1} b(t)>0, \forall t \in \Gamma$. If

$$
\Omega=\bigcup_{t \in \Gamma}\left\{x \in \mathbb{R}^{n} \mid x^{T} B(t) x+2 b(t)^{T} x+c(t) \geq 0\right\},
$$

where $B(t) \in S^{n \times n}, b(t) \in \mathbb{R}^{n}, c(t) \in \mathbb{R}$, and $\Gamma$ is a finite set. Program (1) could be solved by the theory of S-procedure $[9,10]$ which is an important tool for solving programs with quadratic objective functions and quadratic constraints. We could separate program (1) into several parts, and each part is a program with quadratic objective function and quadratic constraints which could be solved by the theory of S-procedure. Then, we could find the global minimum value from all optimal values easily, since $\Gamma$ is finite. But when $\Gamma$ is not a finite set, this method is invalid and we need to find a new method. In this paper, we focus on a special case of program (1), where $\Omega$ has the same structure as program (3). For this special structure, we can try to turn program (1) into a solvable bilevel program.

Some notions and symbols are introduced here. 
Notions and symbols: $\mathbb{R}^{n}$ is the $n$-dimensional real Euler space; $\mathbb{R}^{n \times n}$ denotes the set of all $n \times n$ real matrices; $x_{i}$ is the $i$ th element of $x \in \mathbb{R}^{n} ; S^{n \times n}$ is the subset of $\mathbb{R}^{n \times n}$, which is consisted of the symmetric real matrix; $A>0(A \prec 0)$ and $A \geqslant 0 \quad(A \preccurlyeq 0)$ mean that $A$ is positive definite (negative definite) and positive semidefinite (negative semidefinite), respectively; $I$ is the identify matrix with proper dimension; $e=(1, \ldots, 1)^{T}$; and $\partial f(x)$ is the subgradient of $f(x)$.

\section{Quadratic Program on a Structured Nonconvex Set}

Definition 1 . We call a set $\Omega$ as a structured set generated by $\Gamma$, denoted by $\operatorname{SS}(\Gamma)$, if it satisfies

$$
\Omega=\bigcup_{t \in \Gamma}\left\{x \in \mathbb{R}^{n} \mid x^{T} B(t) x+2 b(t)^{T} x+c(t) \geq 0\right\},
$$

where $\Gamma$ is a subset in $\mathbb{R}^{l}, l<n, B(t) \in S^{n \times n}, B(t) \prec 0$, $b(t) \in \mathbb{R}^{n}, c(t) \in \mathbb{R}$, and $\forall t \in \Gamma$. We call $\Gamma$ as a generator of $\Omega$.

Usually, the dimension of $\Gamma$ is far smaller than the dimension of $\Omega$. $\Gamma$ may be a finite set, such as $\{0,1\}$, a countable infinite set, such as $\mathbb{Z}$, or an uncountable infinite subset of $\mathbb{R}^{n}$. In fact, there are a lot of SS $(\Gamma)$ sets, such as circle, sphere, donut, and crescent. Here are some examples.

\section{Example 1.}

(a) $\left\{x \in \mathbb{R}^{n} \mid-x^{T} x+1 \geq 0\right\} \cup\left\{x \in \mathbb{R}^{n} \mid-x^{T} x+4 e^{T} x+\right.$ $1-4 n \geq 0\}$, where $\Gamma=\{0,1\}, \quad B(t)=-I, \quad$ and $b(t)=\left\{\begin{array}{ll}0 & t=0 \\ 2 e & t=1\end{array}, c(t)= \begin{cases}1 & t=0 \\ 1-4 n & t=1\end{cases}\right.$

(b) $\cup_{t \in \mathbb{Z}}\left\{x \in \mathbb{R}^{n} \mid-x^{T} x+2 t e^{T} x+1-t^{2} n \geq 0\right\}$, where $\Gamma=\mathbb{Z}, B(t)=-I, b(t)=t e$, and $c(t)=1-t^{2} n$

(c) $\cup_{t \in[0.1,1]}\left\{x \in \mathbb{R}^{2} \mid-(1 / t) x^{T} x+\left(\begin{array}{c}2 \\ 2 t\end{array}\right)^{T} x+1-\left(t+t^{3}\right)\right.$ $\geq 0\}$, where $\Gamma=[0.1,1], B(t)=-(1 / t) I, b(t)=\left(\begin{array}{l}1 \\ t\end{array}\right)$, and $c(t)=1-\left(t+t^{3}\right)$.

Example 1 shows that a SS $(\Gamma)$ set is not a convex set in general. (a) is just a union set of two separated spheres, (b) is the union of a series of spheres, and (c) is a horn.

If $\exists t^{*} \in \Gamma$ such that

$$
c\left(t^{*}\right)-b\left(t^{*}\right)^{T} B\left(t^{*}\right)^{-1} b\left(t^{*}\right)<0
$$

then

$$
\left\{x \in \mathbb{R}^{n} \mid x^{T} B\left(t^{*}\right) x+2 b\left(t^{*}\right)^{T} x+c\left(t^{*}\right) \geq 0\right\}
$$

is empty. And if $\exists t^{*} \in \Gamma$ such that

$$
c\left(t^{*}\right)-b\left(t^{*}\right)^{T} B\left(t^{*}\right)^{-1} b\left(t^{*}\right)=0,
$$

then

$$
\left\{x \in \mathbb{R}^{n} \mid x^{T} B\left(t^{*}\right) x+2 b\left(t^{*}\right)^{T} x+c\left(t^{*}\right) \geq 0\right\}
$$

$$
\left\{x \in \mathbb{R}^{n} \mid x=-B\left(t^{*}\right)^{-1} b\left(t^{*}\right)\right\} .
$$

For avoiding the above two simple cases, we make an assumption as follows.

Assumption 1.

$$
c(t)-b(t)^{T} B(t)^{-1} b(t)>0, \quad \forall t \in \Gamma .
$$

We call this assumption as regular assumption.

Now, we consider program (1) with a SS $(\Gamma)$ constraint:

$$
\begin{array}{ll}
\text { inf } & x^{T} A x+2 a^{T} x \\
\text { s.t. } & x \in \bigcup_{t \in \Gamma}\left\{x \in \mathbb{R}^{n} \mid x^{T} B(t) x+2 b(t)^{T} x+c(t) \geq 0\right\} .
\end{array}
$$

We rewrite program (12) into a simpler formulation as follows:

$$
\begin{array}{ll}
\text { inf } & y^{T} A y \\
\text { s.t. } & y \in \bigcup_{t \in \Gamma}\left\{y \in \mathbb{R}^{n} \mid y^{T} B(t) x+2 \widehat{b}(t)^{T} y+\widehat{c}(t) \geq 0\right\},
\end{array}
$$

where

$\widehat{b}(t)=b(t)-B(t) A^{-1} a, \widehat{c}(t)=c(t)-2 b(t)^{T} A^{-1} a+$ $a^{T} A^{-1} B(t) A^{-1} a$, and $y=A^{-1} x+a$.

Then, the following lemma is true.

Lemma 1. If regular assumption holds for program (12), then it still holds for program (13).

Proof.

$$
\begin{aligned}
\widehat{c}(t) & -\widehat{b}(t)^{T} B(t)^{-1} \widehat{b}(t) \\
= & c(t)-2 b(t)^{T} A^{-1} a+a^{T} A^{-1} B(t) A^{-1} a \\
& -\left(b(t)-B(t) A^{-1} a\right)^{T} B(t)^{-1}\left(b(t)-B(t) A^{-1} a\right) \\
& =c(t)-b(t)^{T} B(t)^{-1} b(t)>0 .
\end{aligned}
$$

Now, we just need to focus on program (13).

The following lemma is called as S-Lemma, which presents the relationship between a biquadratic program and a semidefinite program. S-Lemma plays an important role in our analysis.

Lemma 2 (see S-Lemma $[9,10]$ ). If $\left\{x \in \mathbb{R}^{n} \mid x^{T} B x+2 b^{T} x+\right.$ $c \geq 0\}$ has nonempty interior points, then the following two statements are equivalent:

(a) $x^{T} A x+2 a^{T} x+d \geq 0$, for all $x \in\left\{x \in \mathbb{R}^{n} \mid x^{T} B x+\right.$ $\left.2 b^{T} x+c \geq 0\right\}$

(b) there exists $\lambda \geq 0$ satisfying $\left(\begin{array}{cc}A & a \\ a^{T} & d\end{array}\right) \pm \lambda\left(\begin{array}{cc}B & b \\ b^{T} & c\end{array}\right)$

By Lemma 2, for each $t \in \Gamma$, we will find the minimum $d(t)$ such that

$y^{T} A y+d(t) \geq 0, \quad \forall y \in\left\{y \in \mathbb{R}^{n} \mid y^{T} B(t) y+2 \widehat{b}(t)^{T} y+\widehat{c}(t) \geq 0\right\}$,

is just 
first. Then, we solve program $\sup _{t \in \Gamma} d(t)$ to obtain the optimal value of program (13). Summarizing the above discussion, we get the following theorem.

Theorem 1. If regular assumption holds and $A>0$ for program (13), then the optimal value of program (13) is same as that of the following program:

sup $-d$

s.t. $\forall t \in \Gamma, \exists \lambda_{t} \geq 0$ such that $\left(\begin{array}{ll}A & 0 \\ 0 & d\end{array}\right) \pm \lambda_{t}\left(\begin{array}{cc}B(t) & \widehat{b}(t) \\ \widehat{b}(t)^{T} & \widehat{c}(t)\end{array}\right)$.

Proof. If $-d^{*}$ is the optimal value of program (13), then $y^{T} A y \geq-d^{*}, \quad y \in \bigcup_{t \in \Gamma}\left\{y \in \mathbb{R}^{n} \mid y^{T} B(t) y+2 \widehat{b}(t)^{T} y+\widehat{c}(t) \geq 0\right\}$,

which means for all $t \in \Gamma$,

$y^{T} A y+d^{*} \geq 0, \quad y \in\left\{y \in \mathbb{R}^{n} \mid y^{T} B(t) y+2 \widehat{b}(t)^{T} y+\widehat{c}(t) \geq 0\right\}$.

By Lemma 2, for all $t \in \Gamma$, there exists $\lambda_{t} \geq 0$, such that

$$
\left(\begin{array}{cc}
A & 0 \\
0 & d^{*}
\end{array}\right) \geqslant \lambda_{t}\left(\begin{array}{cc}
B(t) & \widehat{b}(t) \\
\widehat{b}(t)^{T} & \widehat{c}(t)
\end{array}\right)
$$

so $d^{*}$ is a feasible point of program (16), and the optimal value of program (13) is not smaller than $-d^{*}$.

On the other hand, if the optimal value of program (16) is $-d^{* *}$; then, for all $t \in \Gamma$, there exists $\lambda_{t} \geq 0$, such that

$$
\left(\begin{array}{cc}
A & 0 \\
0 & d^{* *}
\end{array}\right) \geqslant \lambda_{t}\left(\begin{array}{cc}
B(t) & \widehat{b}(t) \\
\widehat{b}(t)^{T} & \widehat{c}(t)
\end{array}\right)
$$

By Lemma 2, for all $t \in \Gamma$,

$$
y^{T} A y \geq-d^{* *}
$$

for all

$$
y \in\left\{y \in \mathbb{R}^{n} \mid y^{T} B(t) y+2 \widehat{b}(t)^{T} y+\widehat{c}(t) \geq 0\right\},
$$

so

$$
y^{T} A y \geq-d^{* *}
$$

where

$$
y \in \bigcup_{t \in \Gamma}\left\{y \in \mathbb{R}^{n} \mid y^{T} B(t) y+2 \widehat{b}(t)^{T} y+\widehat{c}(t) \geq 0\right\},
$$

which means that $-d^{* *}$ is a low bound of the optimal value of program (13). Hence, the optimal value of program (13) and program (16) are the same.

By Theorem 1, we could solve program (16) instead of solving program (13). Noticing that

$$
\left(\begin{array}{ll}
A & 0 \\
0 & d
\end{array}\right) \geqslant \lambda_{t}\left(\begin{array}{cc}
B(t) & \widehat{b}(t) \\
\widehat{b}(t)^{T} & \widehat{c}(t)
\end{array}\right) \Longleftrightarrow\left(\begin{array}{cc}
A-\lambda_{t} B(t) & -\lambda_{t} \widehat{b}(t) \\
-\lambda_{t} \widehat{b}(t)^{T} & d-\lambda_{t} \widehat{c}(t)
\end{array}\right) \geqslant 0 .
$$

Since $A>0, B(t) \prec 0$, and $\lambda \geq 0$, then

$$
\begin{aligned}
& \left(\begin{array}{cc}
A-\lambda B(t) & -\lambda \widehat{b}(t) \\
-\lambda \widehat{b}(t)^{T} & d-\lambda \widehat{c}(t)
\end{array}\right) \geqslant 0 \\
& \qquad\left\{\begin{array}{l}
A-\lambda B(t) \geqslant 0 \\
\left.d-\lambda \widehat{c}(t)-\lambda^{2} \widehat{b}(t)^{T}(A-\lambda B(t))^{-1} \widehat{b}(t)\right)
\end{array}\right.
\end{aligned}
$$

So program (16) can be rewritten as the following program:

$$
\begin{aligned}
& \text { sup }-d \\
& \text { s.t. } \forall t \in \Gamma,\left\{\begin{array}{l}
\lambda \geq 0 \\
A-\lambda B(t) \pm 0 \\
d-\lambda \widehat{c}(t)-\lambda^{2} \widehat{b}(t)^{T}(A-\lambda B(t))^{-1} \widehat{b}(t) \\
\geq 0 \text { has a solution } \lambda .
\end{array}\right.
\end{aligned}
$$

Noticing that the feasible set of program (27) is just $\forall t \in \Gamma$,

$$
\left\{\begin{array}{c}
\lambda \geq 0, \\
d-\lambda \widehat{c}(t)-\lambda^{2} \widehat{b}(t)^{T}(A-\lambda B(t))^{-1} \widehat{b}(t) \geq 0 \text { has a solution } \lambda,
\end{array}\right.
$$

since $A>0$ and $B(t) \prec 0$. It is still too complex to solve program (27) directly. In the following theorem, we turn program (27) into a bilevel program.

Theorem 2. The optimal value of program (27) is opposite to that of

$$
\sup _{t \in \Gamma} \inf _{\lambda \geq 0} \varphi(\lambda, t)=\lambda \widehat{c}(t)+\lambda^{2} \widehat{b}(t)^{T}(A-\lambda B(t))^{-1} \widehat{b}(t) .
$$

Proof. The infimum $d$ of program (27) satisfies that

$$
\forall t \in \Gamma,\left\{\begin{array}{l}
\lambda \geq 0, \\
d-\lambda \widehat{c}(t)-\lambda^{2} \widehat{b}(t)^{T}(A-\lambda B(t))^{-1} \widehat{b}(t) \\
\geq 0 \text { has a solution } \lambda .
\end{array}\right.
$$

Noticing that for each $t \in \Gamma$, if $d(t)$ is an infimum satisfying (28), then (28) holds for any $d \geq d(t)$. Hence, we just need to find the infimum $d(t)$ satisfying (28) for each $t \in \Gamma$ firstly and then find the supremum $d(t)$ in $t \in \Gamma$.

For each $t \in \Gamma$, we reconsider (28) and let

$$
\begin{gathered}
\varphi(\lambda, t)=\lambda \widehat{c}(t)+\lambda^{2} \widehat{b}(t)^{T}(A-\lambda B(t))^{-1} \widehat{b}(t), \\
\vartheta(t)=\inf _{\lambda \geq 0} \varphi(\lambda, t),
\end{gathered}
$$

then $\vartheta(t)$ must be the infimum $d(t)$ of (28), since if $d(t)<\vartheta(t)$, it is impossible to find $\lambda \in[0,+\infty)$ such that $d(t) \geq \varphi(\lambda, t) \geq \vartheta(t)$; if $d(t)>\vartheta(t)$, by the definition of $\vartheta(t)$, 
S0 Given $\varepsilon>0, \gamma>0, \eta \in(0,1), t^{*} \in \Gamma, k=0$;

S1 Let $\lambda^{k}=0$, compute $\kappa^{k}=(\partial \varphi(\lambda, t)) /\left.\partial \lambda\right|_{\lambda^{k}, t^{*}} ;$ If $\kappa^{k}>0$, then stop and return $\lambda_{k}$;

S2 If $\left|\kappa^{k}\right|<\varepsilon$, then stop and return $\lambda^{k} ;$

S3 Let $\alpha=1$;

S4 If $\varphi\left(\lambda^{k}-\alpha \kappa^{k}, t^{*}\right) \geq \varphi\left(\lambda^{k}, t^{*}\right)-\alpha \gamma\left(\kappa^{k}\right)^{2}$ or $\lambda^{k}-\alpha \kappa^{k}<0, \alpha=\eta \alpha$, goto S4; else $\lambda^{k+1}=\lambda^{k}-\alpha \kappa^{k}, k=k+1$, goto S2;

Algorithm 1: (solving the low-level program).

TAble 1: Numerical results for Algorithm 2.

\begin{tabular}{lccc}
\hline & Time $(\mathrm{s})$ & NOI & Optimal value of (12) \\
\hline$(42)$ & 0.01 & 1 & 0 \\
$(43)$ & 0.07 & 1 & 0.1716 \\
$(44)$ & 0.03 & 5 & 1.2686 \\
$(45)$ & 0.011 & 2 & 1.5069 \\
\hline
\end{tabular}

there must exist $\lambda$ such that $\varphi(\lambda, t) \leq((\vartheta(t)+d(t)) / 2)<d(t)$, which contradicts the definition of $d(t)$. Summarizing the above discussion, we have that the optimal value of program (27) is opposite to that of program (29).

By Theorem 2, we just need to solve program (29). However, program (29) is not easy to solve since it is a sup- inf program. Fortunately, $\varphi(\lambda, t)$ has some good properties.

Property 1.

(a) $\varphi(\lambda, t)$ is a convex function with respect to $\lambda \in[0,+\infty), \forall t \in \Gamma$.

(b) $((\partial \varphi(\lambda, t)) / \partial \lambda)=\widehat{c}(t)+2 \lambda \widehat{b}(t)^{T} M \widehat{b}(t)+\lambda^{2} \widehat{b}(t)^{T} M B$ $(t) M \widehat{b}(t), \forall \lambda \geq 0, t \in \Gamma$, where $M=(A-\lambda B(t))^{-1}$.

(c) If $\widehat{c}(t), \widehat{b}(t)$ and $B(t)$ are differentiable on $\Gamma$, then

$$
\partial \vartheta(t)=\left\{\lambda^{*} \widehat{c}^{\prime}(t)+2\left(\lambda^{*}\right)^{2} \widehat{b}^{\prime}(t)^{T} M \widehat{b}(t)+\left(\lambda^{*}\right)^{3} \widehat{b}(t)^{T} M B^{\prime}(t) M \widehat{b}(t) \mid \lambda^{*} \in \arg \inf _{\lambda \geq 0} \varphi(\lambda, t)\right\}
$$

where $M=\left(A-\lambda^{*} B(t)\right)^{-1}$ and $\vartheta(t)$ is defined in (32).

Proof. (a): $\forall t \in \Gamma$; since $\lambda \geq 0$ and $A-\lambda B(t)>0$, there is an invertible matrix $P$ such that $P^{T} A P=X \pm 0$ and $P^{T}(-B(t)) P=Y>0$, where $X$ and $Y$ are the diagonal matrices. Then,

$$
\lambda^{2} \widehat{b}(t)^{T}(A-\lambda B(t))^{-1} \widehat{b}(t)=\lambda^{2} \widehat{b}^{T}(t) P^{-T}(X+\lambda Y)^{-1} P^{-1} \widehat{b}(t) .
$$

Let $q=P^{-1} \widehat{b}(t)$; then,

$$
\left.\lambda^{2} \widehat{b}(t)^{T}(A-\lambda B(t))^{-1} \widehat{b}(t)\right)=\lambda^{2} \sum_{i=1}^{n} \frac{q_{i}^{2}}{X_{i i}+\lambda Y_{i i}} .
$$

Here,

$$
\frac{\lambda^{2}}{X_{i i}+\lambda Y_{i i}}=\frac{1}{Y_{i i}^{2}}\left(\left(X_{i i}+\lambda Y_{i i}\right)-2 X_{i i}+\frac{X_{i i}^{2}}{X_{i i}+\lambda Y_{i i}}\right)
$$

When $\lambda \geq 0$, every term of the above equation is a convex function, so

$$
\begin{aligned}
& \lambda^{2} \widehat{b}(t)^{T}(A-\lambda B(t))^{-1} \widehat{b}(t), \\
& \lambda \widehat{c}(t)+\lambda^{2} \widehat{b}(t)^{T}(A-\lambda B(t))^{-1} \widehat{b}(t)
\end{aligned}
$$

are convex functions with respect to $\lambda \geq 0$.

(b) and (c): it is obvious from [11].

Remark 1. (a) Since the convexity of $\varphi(\lambda, t)$,

$$
\vartheta(t)=\inf _{\lambda \geq 0} \varphi(\lambda, t)
$$

is an easy solvable program for every $t \in \Gamma$.

(b) If there is $t^{*} \in \Gamma$ satisfying $\widehat{b}\left(t^{*}\right)=0$, then the optimal value of the low-level program is

$$
\inf _{\lambda \geq 0} \varphi\left(\lambda, t^{*}\right)= \begin{cases}0, & \widehat{c}\left(t^{*}\right) \geq 0, \\ -\infty, & \widehat{c}\left(t^{*}\right)<0 .\end{cases}
$$

(c) If regular assumption holds, then $\widehat{c}\left(t^{*}\right)>0$ when $\widehat{b}\left(t^{*}\right)=0$.

(d) $\lambda=0$ is always a feasible point of (38), so

$$
\inf _{\lambda \geq 0} \varphi(\lambda, t) \leq 0, \quad \forall t \in \Gamma
$$

which means that if there exists $t^{*}$ satisfying $\inf _{\lambda \geq 0} \varphi\left(\lambda, t^{*}\right)=0$, then

$$
\sup _{t \in \Gamma} \inf _{\lambda \geq 0} \varphi(\lambda, t)=0 .
$$


S1 Given $\varepsilon>0, \gamma>0, \eta \in(0,1), t^{0} \in \Gamma, k=0$;

S2 Plug $t^{k}$ into the lower-level program to get $\lambda^{k}$;

S3 Compute $\mu^{k} \in \partial \vartheta\left(t^{k}\right)$ and set $\bar{t}_{i}= \begin{cases}u_{i} & \left(\mu^{k}\right)_{i}>0 \\ l_{i} & \left(\mu^{k}\right)_{i}<0, d^{k}=\bar{t}-t^{k} ; \\ \left(u_{i}+l_{i}\right) / 2 & \left(\mu^{k}\right)_{i}=0\end{cases}$

If $\left|\mu_{k}^{T} d_{k}\right|<\varepsilon$, stop and return $\varphi\left(\lambda^{k}, t^{k}\right)$;

S4 Let $\alpha=1$;

S5 If $\theta\left(t^{k}+\alpha d^{k}\right)<\theta\left(t^{k}\right)+\alpha \gamma\left(\mu^{k}\right)^{2}$, then $\alpha=\eta \alpha$, goto S5; else $t^{k+1}=t^{k}+\alpha d^{k}, k=k+1$, goto S2.

Algorithm 2: (solving the upper-level program).

TABLe 2: Numerical results for the discretization method.

\begin{tabular}{lcc}
\hline & Time (s) & Average optimal value of (12) \\
\hline$(42)$ & 0.11 & 0.0110 \\
$(43)$ & 0.23 & 0.1787 \\
$(44)$ & 0.31 & 1.2726 \\
$(45)$ & 0.22 & 1.5311 \\
\hline
\end{tabular}

(e) By the above discussion, if regular assumption holds, and $\exists t^{*} \in T$ such that $\widehat{b}\left(t^{*}\right)=0$, then the value of program (12) is 0 .

\section{Algorithms}

In this section, based on our main result, we present a method for solving program (12) by solving the bilevel program, where we use a linear search method for solving the low-level program and a feasible direction method in [12] for solving the upper-level program. Here, we suppose $T=[a, b]$ (Algorithm 1):

\section{Numerical Results}

In this section, we demonstrate the efficiency and effectiveness of the proposed method by solving some illustrative numerical examples. All of our computations are conducted on a Windows machine, equipped with a dual core $2.4 \mathrm{GHz}$ processor, using MATLAB 7.6 as the computational engine. Noticing that our method does not depend on the dimension of $x$, we just test some examples for $x \in \mathbb{R}^{2}$.

\section{Example 2.}

$$
\begin{array}{ll}
\inf & x^{T} x \\
\text { s.t. } & \cup\{x \in[0.1,1] \\
\cup & \left\{x \in \mathbb{R}^{2} \mid-\frac{1}{t} x^{T} x+\left(\begin{array}{c}
2 \\
2 t
\end{array}\right)^{T} x+1-\left(t+t^{3}\right) \geq 0\right\} .
\end{array}
$$

\section{Example 3.}

$$
\begin{array}{ll}
\text { inf } & x^{T} x \\
\text { s.t. } & \bigcup_{t \in[1,2]}\left\{x \in \mathbb{R}^{2} \mid-x^{T} x+\left(\begin{array}{c}
2 t \\
2 t^{2}
\end{array}\right)^{T} x+t-t^{2}-t^{4} \geq 0\right\} .
\end{array}
$$

Example 4.

$$
\begin{array}{ll}
\text { inf } & x^{T} x \\
\text { s.t. } & \bigcup_{t \in[-1,1]}\left\{x \in \mathbb{R}^{2} \mid\left(x-\left(\begin{array}{c}
\cos (t)+1 \\
t
\end{array}\right)^{T}\left(\left(\begin{array}{ll}
2 & 1 \\
1 & 2
\end{array}\right)+t^{2} I\right)\left(x-\left(\begin{array}{c}
\cos (t)+1 \\
t
\end{array}\right) \leq 1\right\} .\right.\right.
\end{array}
$$

\section{Example 5.}

$$
\begin{array}{ll}
\text { inf } & x^{T} x \\
\text { s.t. } & \bigcup_{t \in[0,2]}\left\{x \in \mathbb{R}^{2} \mid\left(x-\left(\begin{array}{c}
\cos (t)+1 \\
t
\end{array}\right)^{T}\left(\left(\begin{array}{ll}
2 & 1 \\
1 & 2
\end{array}\right)+t^{2} I\right)\left(x-\left(\begin{array}{c}
\cos (t)+1 \\
t
\end{array}\right) \leq 1\right\} .\right.\right.
\end{array}
$$

Computational results for solving the upper-level programs are provided in Table 1, where time represents the average running time in second and NOI represents the number of iterations. $t_{0}$ and err are set as $(l+u) / 2$ and $10^{-5}$, respectively.
We choose the discretization method as the comparing algorithm since there are no other methods for program (12). We select finite different points $t_{i}$ in $\Gamma$ and get their optimal values of 


$$
\begin{array}{ll}
\inf & x^{T} A x+2 a^{T} x \\
\text { s.t. } & x^{T} B\left(t_{i}\right) x+2 b\left(t_{i}\right)^{T} x+c\left(t_{i}\right) \geq 0,
\end{array}
$$

at $t_{i}$, which is equal to the value of

$$
\begin{array}{ll}
\text { sup } & -d \\
& \lambda \geq 0 \\
\text { s.t. } & \left(\begin{array}{ll}
A & a \\
a^{T} & d
\end{array}\right) \geqslant \lambda\left(\begin{array}{ll}
B\left(t_{i}\right) & b\left(t_{i}\right) \\
b\left(t_{i}\right)^{T} & c\left(t_{i}\right)
\end{array}\right) .
\end{array}
$$

Then, we could find out the minimum among these finite number of optimal values to approximate the optimal value of program (12). As for program (47), we use the interior point method [13] or the feasible direction method [12].

Computational results of the discretization method are shown in Table 2, where time represents the average running time in second. For each example, we choose 20 points in $\Gamma$ randomly and test 10 times.

From Table 2, we can see that our method is more effective and efficient than the discretization method.

\section{Conclusion and Future Work}

In this paper, we discuss a quadratic program on a structured nonconvex set. By S-lemma, we convert this program into a bilevel program, where the low-level program is a convex program. Under some assumptions, we develop an algorithm to solve the nonconvex program. Finally, we present the numerical results to show the effectiveness and efficiency of our new method.

Note that we need to solve a lower-level program for each $t_{k}$. However, computational cost of $\left(A-\lambda B\left(t_{k}\right)\right)^{-1}$ is expensive. Hence, how to avoid computing $\left(A-\lambda B\left(t_{k}\right)\right)^{-1}$ is our future work.

\section{Data Availability}

The data used to support the findings of this study are included within the article.

\section{Conflicts of Interest}

The authors declare that they have no conflicts of interest.

\section{Authors' Contributions}

All authors contributed equally and significantly in this paper. All authors read and approved the final manuscript.

\section{Acknowledgments}

This work was supported in part by the National Natural Science Foundation of China (Nos. 11501100, 11571178, 11671082, and 11871149).

\section{References}

[1] E. G. Birgin and J. Mario Martínez, "Large-scale active-set box-constrained optimization method with spectral projected gradients," Computational Optimization and Applications, vol. 23, no. 1, pp. 101-125, 2002.

[2] J. E. Dennis and L. N. Vicente, "Trust region interior point algorithms for minimization problems with simple bounds," in Applied Mathematics and Parallel Computing. Festschrift for Klaus Ritter, H. Fisher, B. Riedmüler, and S. Schäfler, Eds., pp. 97-107, Springer-Verlag, Berlin, Germany, 1996.

[3] J. E. Kelly Jr., "The cutting-plane method for solving convex programs," Journal of the Society for Industrial and Applied Mathematics, vol. 8, no. 4, pp. 703-712, 1960.

[4] Y. Wang, X. Sun, and F. Meng, "On the conditional and partial trade credit policy with capital constraints: a Stackelberg model," Applied Mathematical Modelling, vol. 40, no. 1, pp. 1-18, 2016.

[5] Y. Wang, H. Gao, and W. Xing, "Optimal replenishment and stocking strategies for inventory mechanism with a dynamically stochastic short-term price discount," Journal of Global Optimization, vol. 70, no. 1, pp. 27-53, 2018.

[6] W. W. Hager and H. Zhang, "Recent advances in bound constrained optimization," in International Federation for Information Processing, vol. 199, pp. 67-82, Springer, Berlin, Germany, 2006.

[7] Y. E. Nesterov and M. J. Todd, "Self-scaled barriers and interior-point methods for convex programming," Mathematics of Operations Research, vol. 22, no. 1, pp. 1-42, 1997.

[8] Y. Y. Ye, "Interior point algorithm: theory and analysis," in Series in Discrete Mathematics and Optimization, John Wiley and Sons, New York, NY, USA, 1997.

[9] V. Jeyakumar, N. Q. Huy, and G. Li, "Necessary and sufficient conditions for S-lemma and nonconvex quadratic optimization," Optimization and Engineering, vol. 10, no. 4, pp. 491-503, 2009.

[10] I. Polik and T. Terlaky, "A survey on the S-lemma," SIAM Review, vol. 49, no. 3, pp. 371-418, 2004.

[11] J. F. Bonnans and A. Shapiro, Perturbation Analysis of Optimization Problems, Springer-Verlag, Berlin, Germany, 2000.

[12] Y. Xu and X. Yan, "On a box-constrained linear symmetric cone optimization problem," Journal of Optimization Theory and Applications, vol. 181, no. 3, pp. 946-971, 2019.

[13] R. H. Tutuncu, K. C. Toh, and M. J. Todd, "Solving semidefinite-quadratic-linear programs using SDPT3," Mathematical Programming, vol. 95, no. 2, pp. 189-217, 2003. 